\title{
Diagnosis of Pulmonary Hypertension in Children (Clinical Audit)
}

\author{
GHADA O. MAHMMOD, M.D.; DUAA M. RAAFAT, M.D. and CHRISTEN M. ADEEB, M.Sc. \\ The Department of Pediatrics, Faculty of Medicine, Assiut University, Egypt
}

\begin{abstract}
Background: Patients with suspected PH should be evaluated using a multimodality approach to ensure that they receive a correct diagnosis.

Aim of this study is to measure the appropriateness of the steps of diagnosis pulmonary hypertension in infant and children that has been taken in Cardiology Unit in Assiut University of Children Hospital, against that of international guidelines.
\end{abstract}

Patients and Methods: The study included 60 patients having pulmonary hypertension, data of the patients was collected and analyzed against standard guidelines. Values were expressed in terms of percentages.

Results: Echocardiography, complete blood count and kidney function test were done in all studied cases. Cardiac catheterization was done in only 3 cases $(5 \%)$. The following investigations as CT scan, MRI, $6 \mathrm{MWD}$, pulmonary function test and ventilation/perfusion scan were not done in any case. Coagulation profile was done in 3 cases and other laboratory tests as ANA, thyroid function tests, liver function tests were not done in any case.

Conclusion: Some of investigations took place in $\mathrm{PH}$ diagnosis like (ECG, CXR, echocardigraphy, complete blood count and kidney function test) are considered mandatory investigations in diagnosis of $\mathrm{PH}$. Other investigations like (CT, MRI, 6MWD, pulmonary function test, ventilation/ perfusion scan) and other laboratory tests (coagulation profile, thyroid function tests, ANA,...etc) did not take place in spite of their importance in detecting the cause of $\mathrm{PH}$ as per international guideline.

Key Words: Pulmonary hypertension - Congenital heart disease - Right heart catheterization - Echocardiography.

\section{Introduction}

PULMONARY Hypertension (PH) can present at any age from infancy to adulthood. The distribution

Correspondence to: Dr. Christen M. Adeeb, E-Mail: cocy maher@yahoo.com of etiologies in children is quite different than that of adults, with a predominance of idiopathic pulmonary arterial hypertension and pulmonary arterial hypertension associated with congenital heart disease. In the majority of pediatric patients, $\mathrm{PH}$ is idiopathic or associated with congenital heart disease and rarely is associated with other conditions such as connective tissue or thromboembolic disease [1]

Patients with suspected PH should be evaluated using a multimodality approach to ensure that they receive a correct diagnosis. The series of investigations required includes clinical evaluation, noninvasive imaging techniques and right heart catheterisation (considered to be the "gold standard") for the diagnosis of $\mathrm{PH}$. Current guidelines recommend that a detailed echocardiographic assessment is performed in all patients with suspected $\mathrm{PH}$ [2]

Diagnostic algorithms and implementation of protocols for disease monitoring are desirable to standardize medical care. This leads to a greater insight into disease progression, ultimately to achieve the best possible clinical outcomes [3] .

Algorithm illustrating general diagnostic workup for pediatric pulmonary arterial hypertension. DLCO indicates diffusing capacity of lungs for carbon monoxide; and polysomnography indicates sleep study [4].

\section{The aim of this study is:}

The aim of the work is to measure the appropriateness of the steps of diagnosis pulmonary hypertension in infant and children that did take place or is anticipated in Cardiology Unit in Assiut University Children Hospital, against that of international guidelines. 


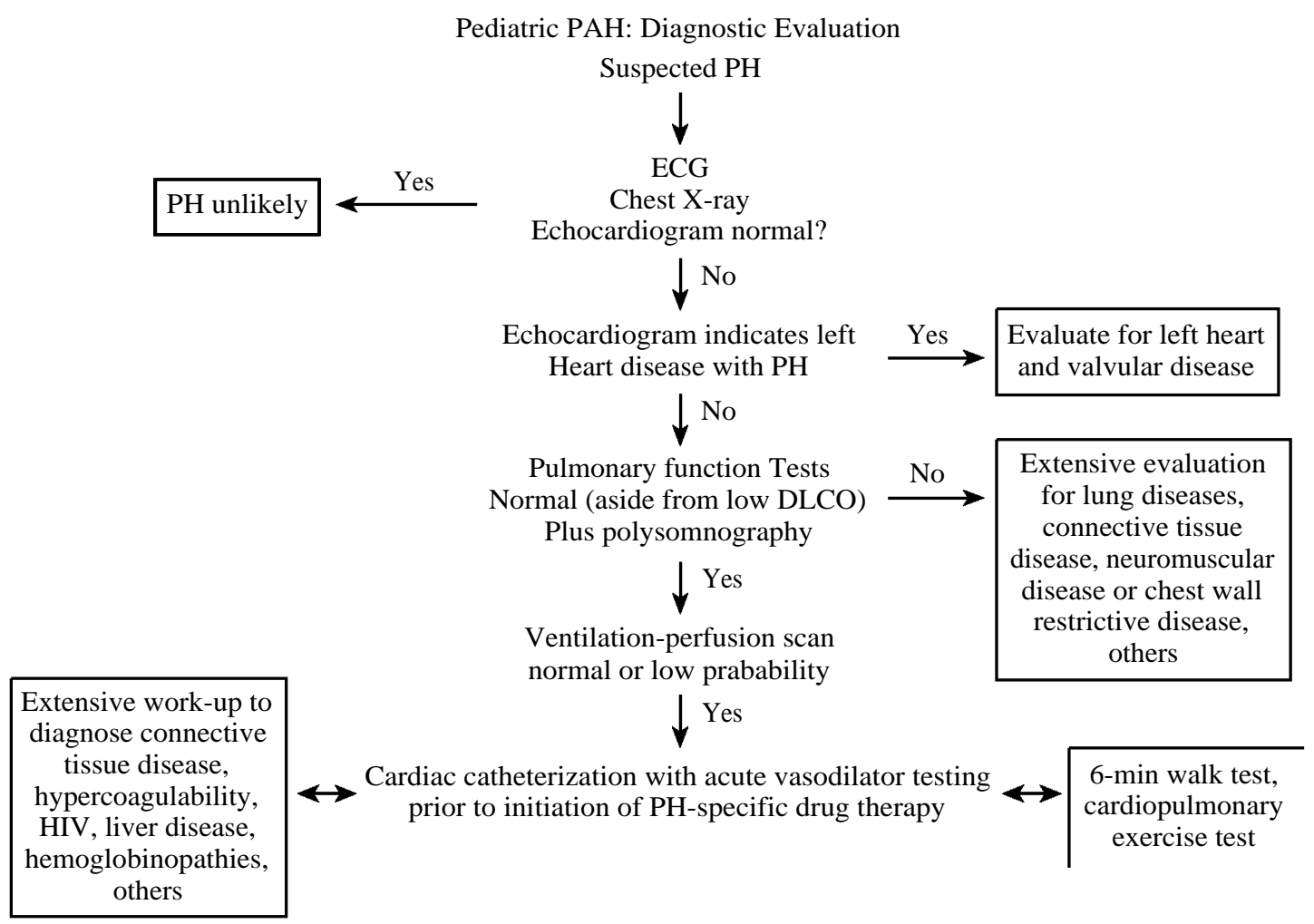

\section{Patients and Methods}

The present study was conducted in Assiut University Children Hospital on all children with PH admitted to Cardiology Unit. The present study included 60 cases of children diagnosed as PH patients above one month of age over one year period from 1 st of February 2016 to 28 February 2017.

Inclusion criteria: All infant and children admitted at Assiut University Children Hospital, Cardiology Unit.

\section{Exclusion criteria: Neonates.}

Data collection: Reviewing sheets of patient with $\mathrm{PH}$ admitted to Assiut University Children Hospital during the study duration.

Ethics consideration and consent: Informed consent was obtained from parents about collection of data, and the research is under the approval of Assiut University Children Hospital, Cardiology Unit.

Following data were collected and recorded for each patient in a master sheet for diagnosis of $\mathrm{PH}$ :

1- Socio-demographic characteristics such as name, age and sex.
2- History of present illness, cyanosis, feeding difficulty, exertional dyspnea, oedema of the lower limbs or puffy eyes, orthopnea $\&$ nocturnal dyspnea, chest pain and recurrent attacks of respiratory symptoms.

3- Prevalence of associated conditions: Congenital heart disease, blood diseases and down syndrome.

4- Vital signs, skin and extremities, chest examination, abdominal examination and heart examination.

5- Investigations such as ECG, chest X-ray, echocardiography and others.

6- Laboratory tests (CBC, KFT and coagulation profile).

\section{Data management and analysis:}

Data of the included patients having pulmonary hypertension were collected and analyzed against standard guidelines. Values were expressed in terms of percentages.

\section{Results}

The study included 60 cases of infant and children diagnosed as $\mathrm{PH}$ who were admitted to the Pediatric Cardiology Department at Assuit University Children Hospital over one year period from 1 st of February 2016 to 28 February 2017. 
Most of the cases 40 cases $(66.7 \%)$ fall in the age group < 1 year, followed by 12 cases $(28 \%)$ were from 5 to 16 years.

The vast majority of the studied cases 50 cases $(83.3 \%)$ had history of recurrent attacks of respiratory symptoms followed by exertional dyspnea 40 cases $(66.7 \%)$. Equal proportion were with feeding difficulty 37 cases $(61.7 \%)$. Cyanosis was found in 22 cases (36.7\%). Only 11 cases (18.3\%) had oedema of the lower limbs or puffy eyes and 11 cases $(18.3 \%)$ had orthopnea. Lastly chest pain was reported in 11 cases $(18.3 \%)$ while data were not recorded in 9 cases $(15 \%)$ and not applicable in 40 cases $(66.7 \%)$.

Congenital heart disease was the highest associated condition in 48 cases $(80 \%)$. This is followed by 10 cases $(16.7 \%)$ whom had associated with down syndrome. On the other side only 1 case $(1.7 \%)$ presented with blood disease in the form of Thalasaemia Major.

Fifty three cases $(88.3 \%)$ had high temperature. Tachycardia was presented in 41 cases $(68.3 \%)$. Tachypnea was found in 27 cases (45\%). Hypotension was found in 11 cases $(18.3 \%)$.

Chest examination was done in all studied cases. The majority of the cases 31 cases $(51.7 \%)$ had adventitious sound in the form of creptations. Respiratory distress was documented in 27 cases $(45 \%)$.

Abdominal examination was done in all studied cases. Nothing was found in the majority of cases 46 cases $(76.3 \%)$ followed by hepatomegaly which was found in 13 cases (21.7\%). Splenomegaly was found in one patient who had thalassemia major.

Fig. (1) shows cardiac examination (inspection, palpation and auscultation) in the studied cases. By inspection 49 cases $(81.7 \%$ ) were normal. By palpation the apex were shifted outside the 5 th midclavicular line in 44 cases $(73.3 \%)$. By auscultation most of the cases 47 cases $(78.3 \%$ ) had accentuated second heart sound followed by ejection systolic murmur which was heard in 32 cases $(53.3 \%)$.

Table (1) shows ECG of all studied cases with the following results: The highest finding in the studied cases was tachycardia in 41cases $(68.3 \%)$. Then p_pulmonale was found in 33 cases $(55 \%)$. Only 14 cases $(23.3 \%)$ had right axis deviation in their ECG.
Fig. (2) shows that chest X-ray findings of all studied cases. In the vast majority of the studied cases 53 cases $(88.3 \%)$ their chest X-ray showed bronchopnemonic patches. Also cardiomegaly was found in 41 cases $(68.3 \%$ ) and signs of pulmonary congestion were found in 38 cases $(63.3 \%)$.

Fig. (3) shows echocardiography findings of all studied cases. All cases (100\%) showed tricuspid regurge followed by 40 cases $(66.7 \%)$ who showed right sided dilatation. Then 20 cases $(33.3 \%)$ showed pulmonary regurge and 16 cases $(26.7 \%)$ showed pulmonary artery dilatation. Only D shaped $\mathrm{RV}$ was found in 11 cases (18.3\%).

Table (2) shows cardiac catheterization was done in only 3 cases (5\%). On the other side the following investigations CT scan, MRI, 6 MWD, pulmonary function test and ventilation/perfusion scan were not done in any case. Overall studied cases had complete blood count and kidney function test. Coagulation profile was done in 3 cases as pre-operative investigation before cardiac catheterization not as apart for diagnosis of PH. Other laboratory tests (ANA, thyroid function tests and liver function tests) were not done in any of the studied cases.

Table (1): ECG finding of the studied cases.

\begin{tabular}{lcccc}
\hline \multirow{2}{*}{ Investigation } & \multicolumn{2}{c}{ Done } & \multicolumn{2}{c}{ Not done } \\
\cline { 2 - 5 } & No. & $\%$ & No. & $\%$ \\
\hline ECG & 60 & 100.0 & 0 & 0.0 \\
Tachycardia: & & & 0 & 0.0 \\
$\quad$ Yes & 41 & 68.3 & 0 & 0.0 \\
$\quad$ No & 19 & 31.6 & 0 & 0.0 \\
$P$-pulmonale: & & & 0 & 0.0 \\
Yes & 33 & 55.0 & 0 & 0.0 \\
$\quad$ No & 27 & 45.0 & 0 & 0.0 \\
Rt axis deviation: & & & 0 & 0.0 \\
$\quad$ Yes & 14 & 23.3 & 0 & 0.0 \\
$\quad$ No & 46 & 76.7 & 0 & 0.0 \\
\hline
\end{tabular}

Table (2): Data about other investigations in studied cases.

\begin{tabular}{lcccc}
\hline \multirow{2}{*}{ Investigation } & \multicolumn{2}{c}{ Done } & \multicolumn{2}{c}{ Not done } \\
\cline { 2 - 5 } & No. & $\%$ & No. & $\%$ \\
\hline Catheterization & 3 & 5.0 & 57 & 95.0 \\
CT & 0 & 0.0 & 60 & 100.0 \\
MRI & 0 & 0.0 & 60 & 100.0 \\
Sleep study: & & & & \\
$\quad$ 6 MWD & 0 & 0.0 & 60 & 100.0 \\
$\quad$ Pulmonary function test & 0 & 0.0 & 60 & 100.0 \\
$\quad$ Ventilation/perfusion scan & 0 & 0.0 & 60 & 100.0 \\
Laboratory test: & & & & \\
$\quad$ Complete blood count & 60 & 100.0 & 0 & 0.0 \\
$\quad$ Kidney function test & 60 & 100.0 & 0 & 0.0 \\
$\quad$ Coagulation profile & 3 & 5.0 & 57 & 95.0 \\
\hline
\end{tabular}




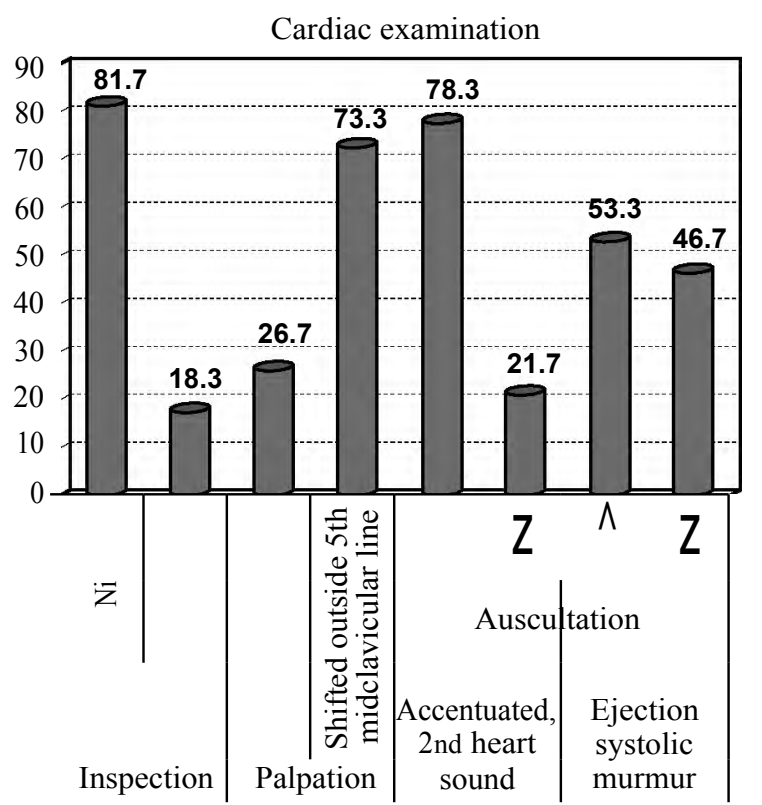

Fig. (1): Examination of heart of the studied cases.

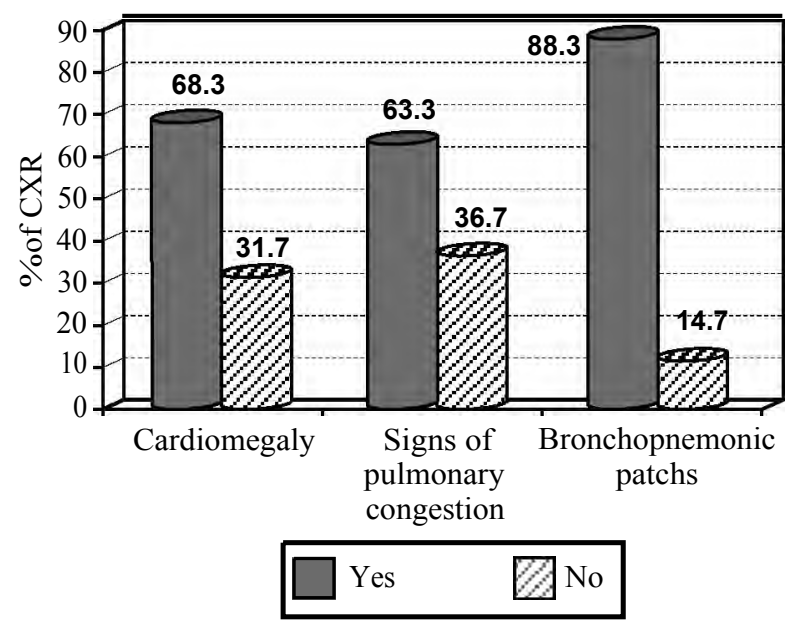

Fig. (2): Chest X-ray findings of the studied cases.

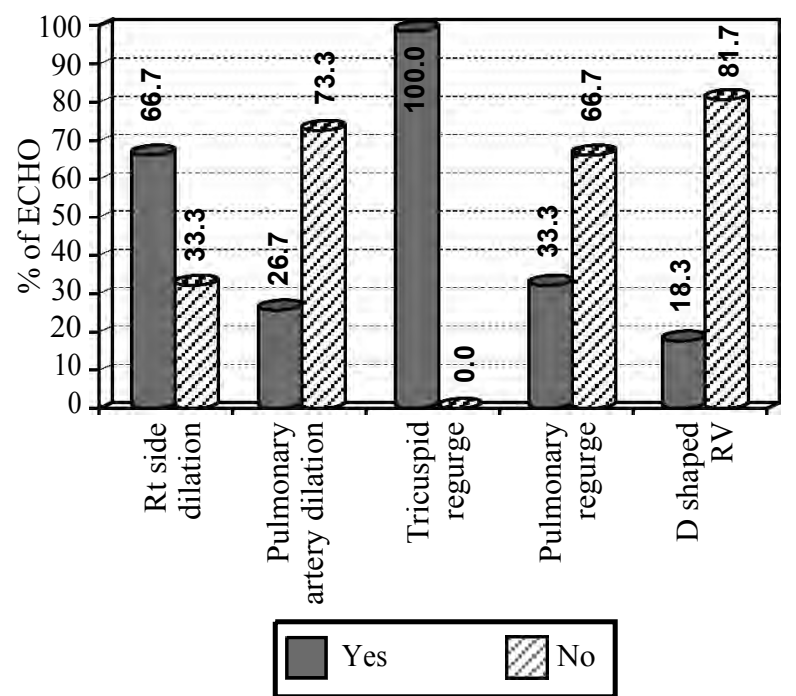

Fig. (3): Echocardiography findings of the studied cases.

\section{Discussion}

In this study the vast majority 50 cases $(83.3 \%)$ were with history of recurrent attacks of respiratory symptoms followed by exertional dyspnea 40 cases (66.7\%) which considered to be one of the main presenting symptoms and 37 cases $(61.7 \%)$ were with feeding difficulty. Where as, cyanosis was found in 22 cases $(36.7 \%)$. However, much less cases 11 cases $(18.3 \%)$ were found with oedema of the lower limbs or puffy eyes, orthopnea $\&$ nocturnal dyspnea as they are considered manifestations of right sided heart failure. Lastly chest pain was reported in 11 cases $(18.3 \%)$ while data were not found in 9 cases $(15 \%)$ of cases and not applicable in 40 cases $(66.7 \%)$ this may be due to the majority of the studied cases were in the age group $<1$ year. This results are concomitant with the study Ali, et al., 2016 [5] .

Kozlik-Feldmann et al., 2016 [6] stated that the most common type of PH diagnosed in childhood is PAH associated with CHD also in our study the vast majority of the diagnosed $\mathrm{PH} 48$ cases $(80 \%)$ were CHD.

Down syndrome cases are more susceptible to PH (Cerro et al., 2015) as 10 of our cases (16.7\%) were Down syndrome [4].

One case $(1.7 \%)$ presented with blood disease in the form of thalasaemia major as chronic hemolytic anemia such as sickle cell disease and thalassemia, are associated with an increased risk of $\mathrm{PH}$ as discussed in the recent study by Simonneau et al., 2013 [7].

Regarding examination of skin and extremities; the following findings: Central cyanosis was found in 22 cases $(36.7 \%)$ as explained by Michael et al., 2004 study [8].

In a study was done by Galie et al., 2016 digital clubbing was encountered in cyanotic CHD and interstitial lung disease or liver disease. Clubbing of fingers was found in 20 of our cases $(33.3 \%)$. While 11 cases $(18.3 \%)$ showed oedema and raised jugular venous pressure was found in 9 cases $(15 \%)$. Only one case $(1.7 \%)$ showed jaundice which was found in association with patient with thalassaemia and $\mathrm{PH}$ [9]

Chest examination revealed that 31 cases $(51.7 \%)$ had creptations and respiratory distress happened in 27 cases $(45 \%)$. The same was observed in the study Michael et al., 2004 [8]

In our study hepatomegaly was found in 13 cases $(21.7 \%)$. The least finding was splenomegaly 
found in only one case $(1.7 \%)$ who had thalassaemia major. The study of Michael et al., 2004 showed that hepatomegaly is indicative of right ventricular failure or presence of blood disease [8]

Several clinical signs may be appreciated upon examination and auscultation of the heart. In this study cardiac examination was applied for all studied cases. By inspection 49 cases $(81.7 \%)$ were normal. By palpation the apex were shifted outside the 5 th midclavicular line in 44 cases $(73.3 \%)$. By auscultation 47 cases $(78.3 \%$ ) had accentuated second heart sound followed by ejection systolic murmur heard in 32 cases $(53.3 \%$ ). Similar results were discussed by Michael et al., 2004 as an accentuated pulmonary component of the second heart sound audible at the apex reflects increased force of pulmonary valve closure due to $\mathrm{PH}$ and a murmur is due to sudden interruption of pulmonary valve opening $[8]$.

Qureshi et al., 2017 suggeted that baseline investigations should include chest X-ray, ECG and transthoracic echocardiography and all these investigations were fulfilled in our study [10]

Our study shows ECG findings of PH in form of ppulmonale which was found in 33 cases (55\%) and 14 cases $(23.3 \%)$ had right axis deviation in their ECG.

The study was done by Ali et al., 2016, discussed ECG manifestations of PH correspond to increased right-sided heart strain and overload [5]

Chest X-ray was done in all cases of this study. According to Ali et al., 2016, chest X-ray is usually the initial imaging study performed [5]

Echocardiography was done for all cases in this study. All cases showed tricuspid regurge followed by 40 cases $(66.7 \%)$ who showed right sided dilatation. Then 20 cases $(33.3 \%)$ showed pulmonary regurge and 16 cases $(26.7 \%)$ showed pulmonary artery dilatation. Only D shaped RV was found in 11 cases $(18.3 \%)$. The importance of echocardiography was illusterated by Qureshi et al., 2017 [10] .

In this study, the following investigations CT scan, MRI, $6 \mathrm{MWD}$, pulmonary function test and ventilation/perfusion scan were not done in any case in spite of their importance in diagnosis and detecting the cause of $\mathrm{PH}$ and this may be due to their unavailability all the time. Cardiac catheterization was done in only 3 cases (5\%) this may be due to its invasiveness and being not following the international guideline. Only CBC and kidney function test were done in all cases. Coagulation profile was done in 3 cases (5\%) as part of preparation for cardiac catheterization and not as part of diagnostic tests. Laboratory tests (hepatic profile and autoimmune panel) were not done in any case. This is against the study of Trenton and Steven, 2001 and the international guidelines which discussed the importance of every investigation to reach the accurate diagnosis and plan the strategy of treatment of $\mathrm{PH}$ in pediatric [11].

\section{Recommendations:}

To obtain accurate diagnosis, minimize morbidity and mortility of $\mathrm{PH}$ and proper treatment we recommended the following:

1- The diagnosis of pulmonary hypertension can be made on echocardiography, but a multitude of investigations may need to be done to narrow down a cause.

2- In patients with a clinical suspicion of $\mathrm{PH}$, echocardiography should be performed to evaluate the level of right ventricular systolic pressure, and to assess the presence of associated anatomic abnormalities such as tricuspid regurge, pulmonary regurge, right atrial enlargement and right ventricular enlargement.

3- The following investigations CT scan, MRI, 6 MWD, pulmonary function test and ventilation/ perfusion scan should be done due to their important role in diagnosis and detecting the cause of $\mathrm{PH}$.

4- The Right Heart Catheterization (RHC) is the gold standard test to diagnose and characterize $\mathrm{PH}$. The RHC testing to confirm the diagnosis of PAH and direct treatment decision should be performed at expert centers.

5- All patients with suspected or confirmed PH should undergo serological routine hematologic and biochemical analysis as well as testing for connective tissue diseases, chronic liver disease, and thyroid function.

\section{References}

1- DUNBAR D., STEVEN H., ROBYN J., et al.: Pediatric Pulmonary Hypertension. Journal of the American College of Cardiology Vol. 62, No. 25, Supply D, 2013.

2- LUKE S., JULIA G., DAVID D., et al.: Echocardiographic assessment of pulmonary hypertension. European Respiratory Review, 21: 125, 239-48, 2012.

3- KOESTENBERGER M., APITZ C., ABDUL-KHALIQ H., et al.: Transthoracic echocardiography for the evaluation of children and adolescents with suspected or confirmed pulmonary hypertension. The European paediatric pulmonary vascular disease network, endorsed by ISHLT and DGPK. Heart, 102: ii14-22, 2016. 
4- CERRO M.J., ABMAN S., DIAZ G., et al.: A consensus approach to the classification of pediatric pulmonary hypertensive vascular disease: Report from the pediatric taskforce, panama 2011. Pulmonary Circulation, 1: 28698, 2011.

5- ALI A., SHEYLAN P., JESSICA C., et al.: Pulmonary arterial hypertension and associated conditions. Diseasea-Month; November 2016 Volume 62, Issue 11, Pages 382-405, 2016.

6- KOZLIK-FELDMANN R., HANSMANN G., BONNET D., et al.: Pulmonary Hypertension in Children and Adolescents with Congenital Heart Disease. Heart, 102: ii428, 2016.

7- SIMONNEAU G., GATZOULIS M.A., ADATIA I., et al.: Updated clinical classification of pulmonary hypertension. J. Am. Coll. Cardiol., 62 (Suppl): D34-41, 2013.

8- MICHAEL M., DAVID G., VIRGINIA S., et al.: Screen- ing, early detection, and diagnosis of pulmonary arterial hypertension: ACCP evidence-based clinical practice guidelines. Chest, 126: 14S-34S, 2004.

9- GALIE N., HUMBERT M., VACHIERY J.L., et al.: Authors/Task Force M. 2015. ESC/ERS guidelines for the diagnosis and treatment of pulmonary hypertension: The joint task force for the diagnosis and treatment of pulmonary hypertension of the European Society of Cardiology (ESC) and the European Respiratory Society (ERS). European Heart J., 37: 67-119, 2016.

10- QURESHI A.Z. and TULLOH R.M.R.: Pediatric pulmonary hypertension: Aetiology, pathophysiology and treatment, Paediatrics and Child Health, Volume 27, Issue 2, February, Pages 50-57, 2017.

11-TRENTON D. and STEVEN W.: Diagnosis and treatment of pulmonary hypertension. Am. Fam. Physician., 63: 1789-98, 1800, 2001.

\section{تشخيص إرتفاع الضغط الرئوى فى الآطفال (دراسة تدقيقية)}

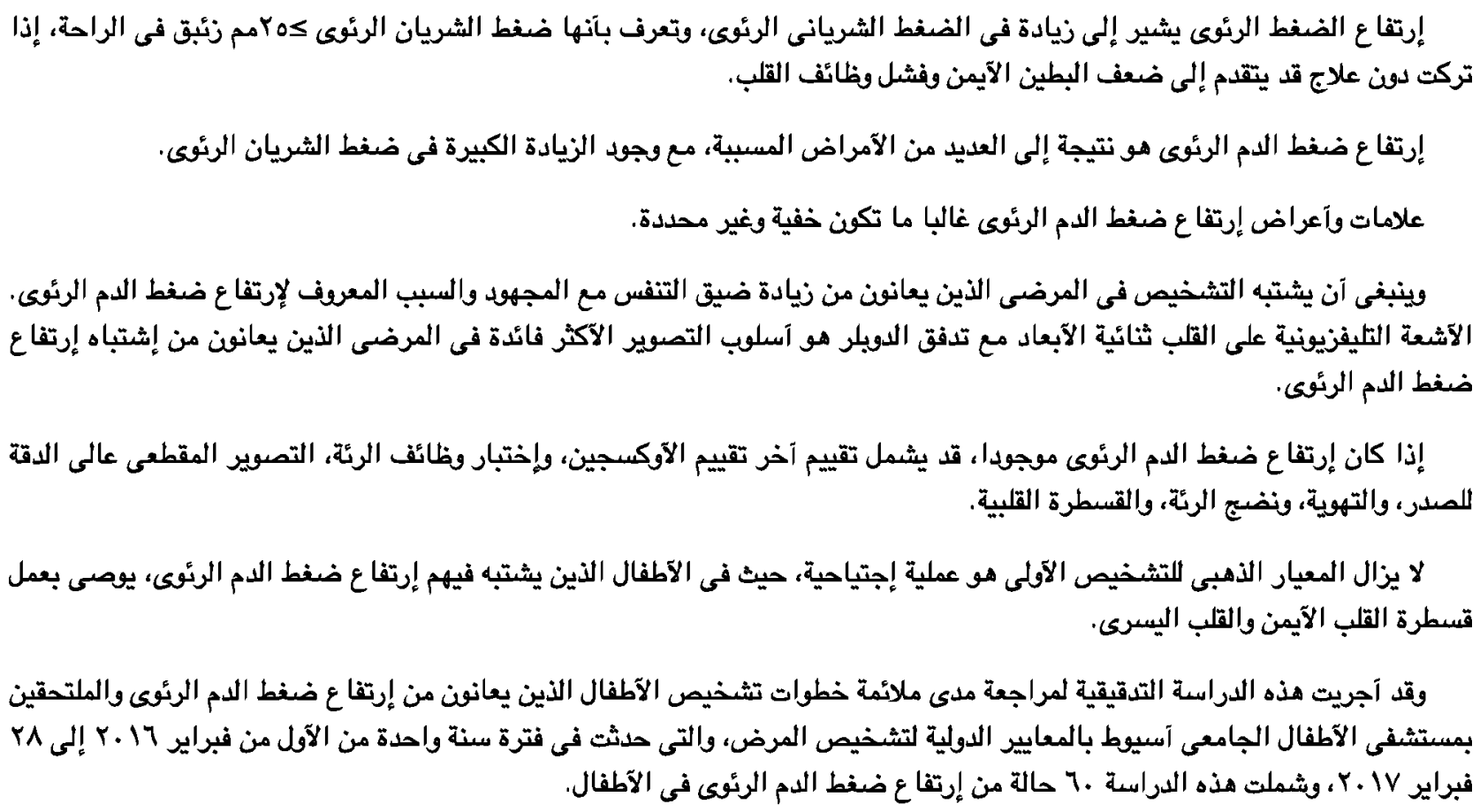

